\title{
Find Out Pen Drive Location with the Help of Mobile GPS
}

\author{
Suhas Machhindra \\ Gaikwad \\ Symbiosis Institute of \\ Technology (SIT), \\ Pune Affiliated to \\ Symbiosis International \\ University (SIU), Pune
}

\author{
Rahul Joshi \\ Symbiosis Institute of \\ Technology (SIT), \\ Pune Affiliated to Symbiosis \\ International University (SIU), \\ Pune
}

\author{
Shishir Machhindra \\ Gaikwad \\ Modern college, Progressive \\ Education Society's \\ Shivajinar, Pune 411005
}

\begin{abstract}
Paper will give a systematic idea of finding out pen drive location with the help of mobile GPS. It will require a circuitcontaining sensor will give position in terms of latitude and longitude. Now place Barcode on the pen drive so that pen drive will have certain number is used to identify that pen drive. Barcode placed on that pen drive is read using the mobile camera act as Barcode scanner here onwards role of GPS will take place as pen drive have latitude and longitude with pen drive id numbers from Barcode is known then add the Barcode functionality in the GPS so that the GPS will read barcode store numbers and will get the latitude and longitude.
\end{abstract}

\section{General Terms}

Mobile GPS, Pen Drive et.al.

\section{Keywords}

Barcode reader, sensor circuit, Mobile GPS

\section{INTRODUCTION}

Transfer of data from one system to another is made easy with the help of pen drive. Pen drive is used to transfer large amount of data from one machine to another and made easy to transfer of data.

Pen drive is a small device because of its small size it could be lost somewhere else and might have the possibility that it will not find. If pen drive containing a very useful information and by user it may be lost else here come the necessity of finding pen drive $[1,2,3,4,5,6,7]$. The job of finding any place, street or address is now made very easy with the help of mobile GPS $[8,9,10]$. Just enter the address or pin code of that location GPS will convert address to latitude and longitude and will shows the path how to reach that particular address $[15,16,17,18]$. Now how the GPS works, there are 24 satellite above the earth's surface for GPS which will give the position of any object for find each satellite will make circular orbit of its own to find out the position of each object there is at least four satellite is required $[19,20,21,22]$. Each satellite has its own orbit when four orbits of satellite will cross each other at certain points they will location of any object in terms of latitude and longitude [23,24].

\section{METHODOLOGY}

Design a circuit in such manner that it will give location of pen drive. Circuit containing the main components is represented in the block diagram (1).

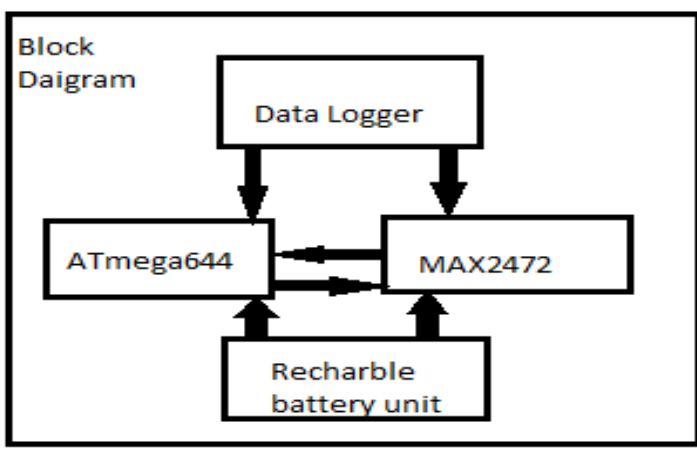

Figure 1: Block Diagram of proposed work

a) ATmega644

ATmega644 is responsible for the transfer of data between all hardware components of the circuit.

\section{b) $\operatorname{Max} 2472$}

Max2472 is used for tracing the location to find out the latitude and longitude position of the device or any object.

\section{c) Data logger}

Data logger used here for the purpose of storing all the data collected in the form of latitude and longitude will be sent to a data logger and data logger will save that data.

\section{d) Rechargeable unit}

Now supply power to the circuit a rechargeable unit is used, this rechargeable unit will supply the power to the circuit it is a USB rechargeable unit it will be recharged if pen drive is connected to the laptop.

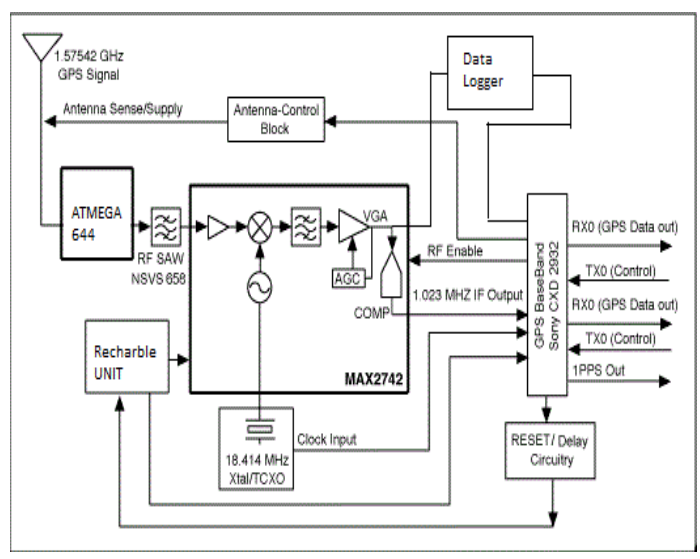

Figure 2: Circuit diagram for developed model 
In the spherical coordinate need to solve Laplace's equation in spherical coordinates

$\frac{1}{r} \frac{d^{2}}{d r^{2}}\left(r^{*} v\right)+\frac{1}{r^{2} * \sin \theta} \frac{d}{d \theta}\left(\sin \theta \frac{d v}{d \theta}\right)+\frac{1}{r^{2} * \sin \theta} \frac{d^{2} v}{d \lambda^{2}}=0$

Solution to gravity of potential in spherical coordinates can be solved by separation of variables $r$ =radius, $\square=$ longitude $\square$ $=$ co latitude

$v(r, \theta)=R(r) * g(\theta) * h(\lambda)$

Now consider each satellite has to calculate the position of pen drive so there will be minimum four satellites will be needed to calculate the location of pen drive so each satellite has three unknown $\mathrm{x}, \mathrm{y}, \mathrm{z}$ and $\mathrm{d}$ is the distance from the satellite to the GPS receiver circuit equation can be represented by

$$
\begin{aligned}
& \left(x-x_{1}\right)^{2}+\left(y-y_{1}\right)^{2}+\left(z-z_{1}\right)^{2}=d_{1}{ }^{2} \\
& \left(x-x_{2}\right)^{2}+\left(y-y_{2}\right)^{2}+\left(z-z_{2}\right)^{2}=d_{2}{ }^{2} \\
& \left(x-x_{3}\right)^{2}+\left(y-y_{3}\right)^{2}+\left(z-z_{3}\right)^{2}=d_{3}^{2} \\
& \left(x-x_{4}\right)^{2}+\left(y-y_{4}\right)^{2}+\left(z-z_{4}\right)^{2}=d_{4}{ }^{2}
\end{aligned}
$$

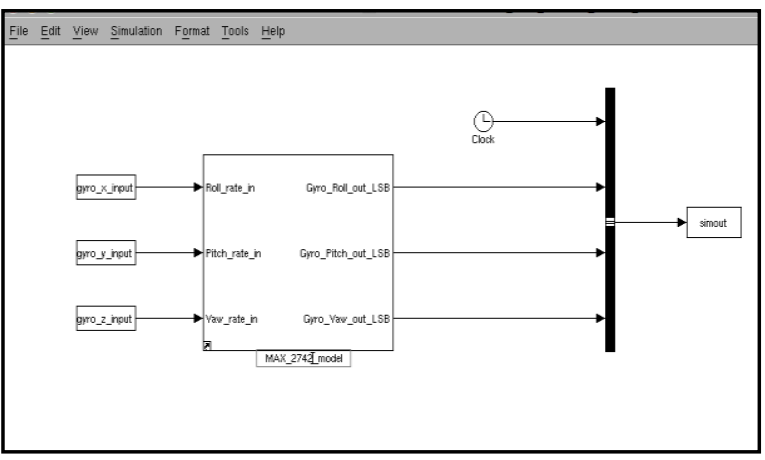

Figure 3: Simulink model for proposed work

With the distance from satellite $d$ is known equation can be converted to three equations and three unknown $\mathrm{x}, \mathrm{y}$ and $\mathrm{z}$. Therefore, circuit is modeled in Matlab, Simulink fig (3) with MAX2742 model takes an input as yaw, pitch and roll around the three different axis expressed in degrees and as a result of simulation the produced in the digital form LSBit unit

Model is represented as a sensor element frequency response and internal digital filtering are modeled internal analog and digital saturation was eliminated.

However use Barcode, which can place near the circuit in fig (4)

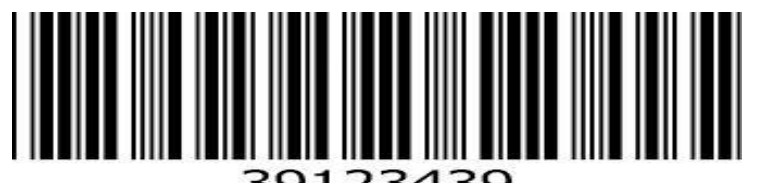

Figure 4: Barcode representation of code
Barcode has specific number is attached to the pen drive so that Barcode will give a unique identity number to the pen drive. Therefore, pen drive now has fixed identity number and circuit gives position in terms of latitude and longitude. However to read Barcode placed on pen drive Mobile camera is used as a barcode scanner in fig (5) .the particular Barcode placed on the pen drive can be read easily

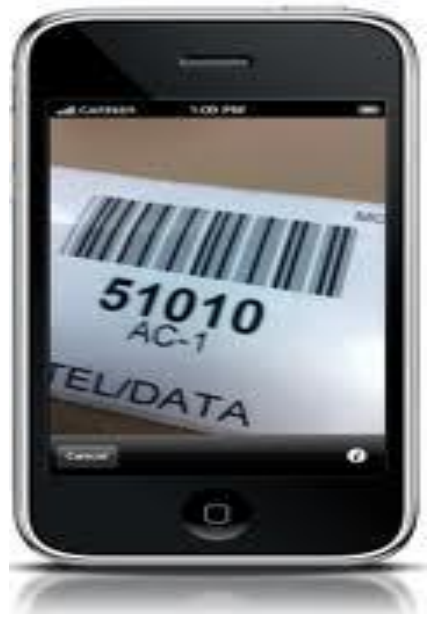

Figure 5: Mobile camera works as Barcode reader

In the mobile GPS now required adding the functionality of Barcode reader so that mobile GPS will read any Barcode situated on pen drive it will be displayed in fig (6).

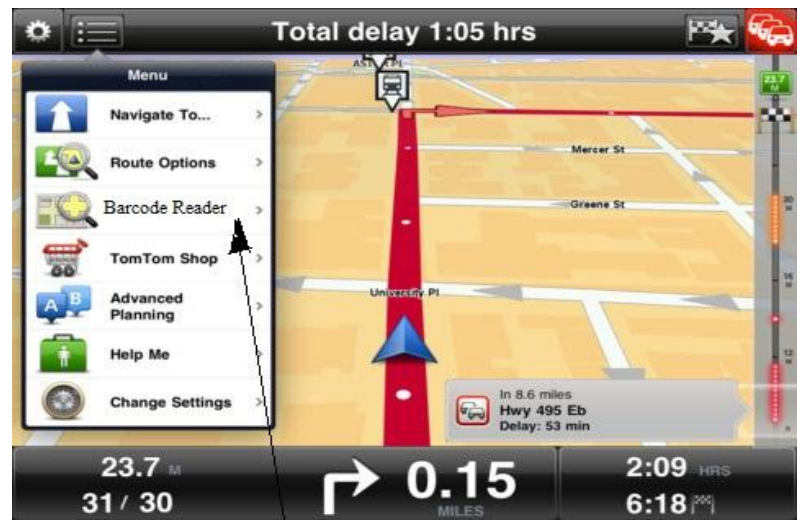

Figure 6: Mobile GPS with Barcode reader functionality

GPS will support the different development environment like Microsoft visual $\mathrm{c}++$ and visual basic and visual $\mathrm{c}$ shaft here by using any programming language Barcode reader function will be added. In order to use GPS toolkit from visual c++ need to add files to new project they are

\#include "GpsCtrl.h"

\#include "GpsCtrl_i.c"

\#include "GpsConstantX.h"

However declare the component as

Private objGps

As Gps

Private objBarcodereader

As Barcode reader

Private objGpsUtilities

As GpsUtilities

Private objGpsDatumParameters As GpsDatumParameters

Private objGpsGridParameters As GpsGridParameters 
Private objGpsConstant

As objGpsConstant

Now should able to create an instance of the object

objGps =new GPS();

ObjBarcodereader=new objBarcodereader ();

ObjGpsUtilities=new objGpsUtilities ();

ObjGpsDatumParameters=new objGpsDatumParameters ();

ObjGpsGridParameters=new objGpsGridParameters ();

ObjGpsConstant =new objGpsConstant ();

Code sample of GpsDatum Parameter object to configure is

Sim objDatum

Set objDatum=CreatObject (“Ab. GpsDatumParameters”)

ObjDatum.clear

$\begin{array}{lc}\text { ObjDatum. Barcodereader } & =39123429 \\ \text { ObjDatum. TranslationX } & =446.448 \\ \text { ObjDatum. TranslationY } & =-125.448 \\ \text { ObjDatum. TranslationZ } & =542.448 \\ \text { ObjDatum. RotationX } & =0.150 \\ \text { ObjDatum. RotationY } & =0.247 \\ \text { ObjDatum. RotationZ } & =0.842\end{array}$

ObjDatum. SaveTofile "c:|Datums $\backslash O S G B 36 . i n i ”$

By using this approach mobile GPS can be equipped with the Barcode reader functionality.

Therefore mobile GPS is all equipped with the Barcode reader function to use in full throttle.

\section{RESULTS}

Matlab Simulink of the model is given will generate the result

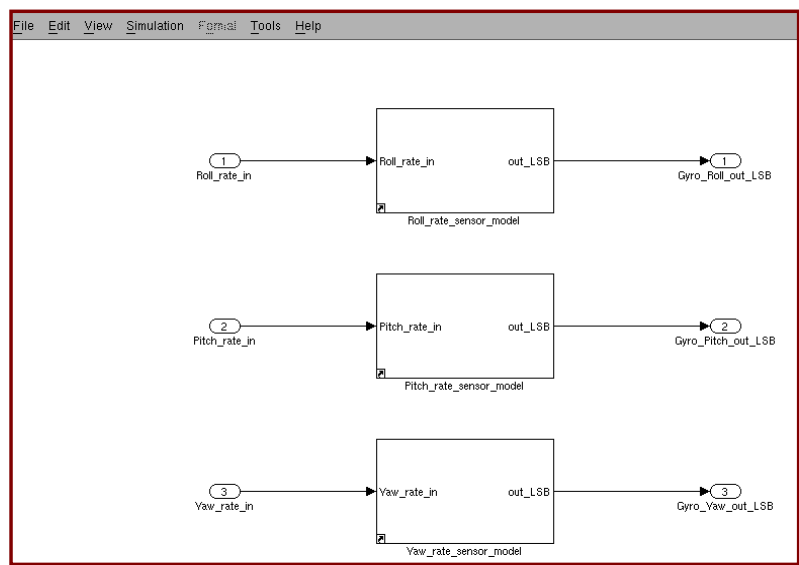

Figure 7: Simulink model for proposed work

This will produce with MAX2742 model takes an input as yaw, pitch and roll around the three different axis expressed in degrees and as a result of simulation the product in the digital form LSBit unit

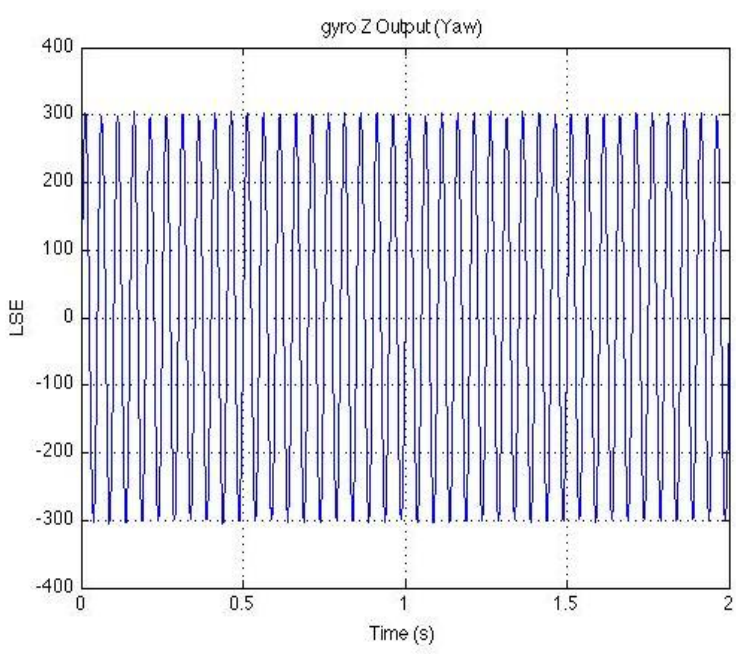

Figure 8: Output in digital form LSBit unit of Z-axis representing Yaw.

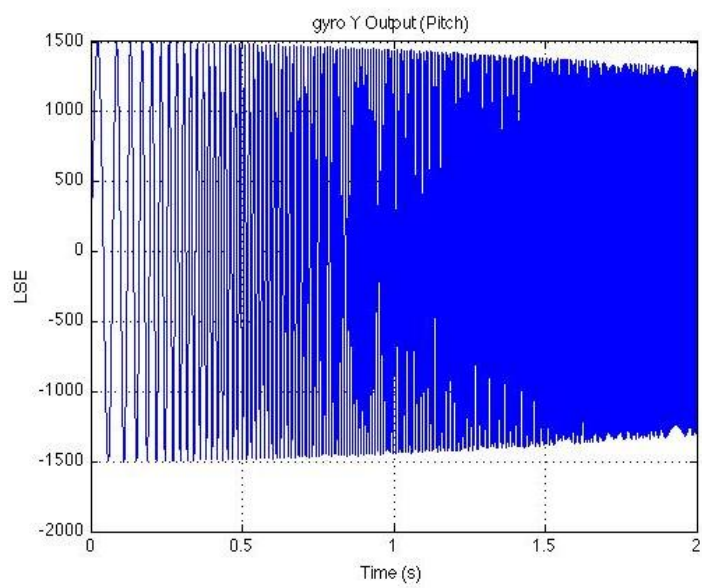

Figure 9: output in digital form LSBit unit of $Y$ axis representing Pitch.

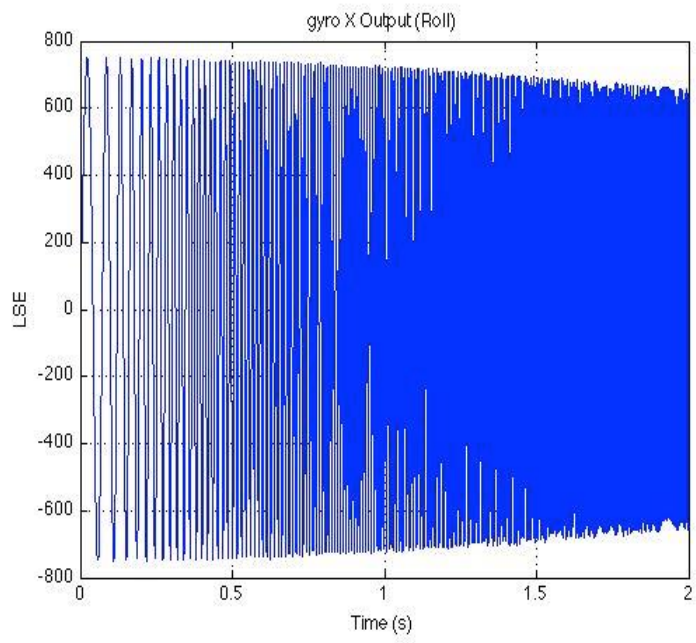

Fig 10: output in digital form LSBit unit of $X$ axis representing Roll.

Output generated here is in digital form LSBit unit of three different axis is representing the position of pen drive with respect to $\mathrm{X}, \mathrm{Y}, \mathrm{Z}$ axis 


\section{Limitation for locating pen drive}

There are 24 satellites above the earth's surface for GPS, which will give the position of pen drive placed anywhere above the earth's surface if pen drive is kept in book as book marker there is no issue in locating pen drive position or kept in car may be lost in train or pocket of jacket it is an easy to locate pen drive.

\section{CONCLUSION}

In this work proposed an idea of locating the pen drive and implemented with the help of circuit designed and implementation of circuit is shown in Matlab Simulink model which gives results and added functionality in GPS for Barcode which make easy for finding job of any object.

\section{FUTURE WORK}

Future work will base on locating pen drive without using any kind of sensor or circuit placed on pen drive it is done by using with the help of only mobile GPS.

\section{ACKNOWLEDGMENTS}

Our thanks to the experts who have contributed towards development of the project that is Anita Machhindra Gaikwad, Shishir Machhindra Gaikwad, Sayali Machhindra Gaikwad.

\section{REFERENCES}

[1] Diana Berbecaru Politecnico di Torino, Dip. di Automatica e Informatica Corso Duca degli Abruzzi "19th International Euro micro Conference on Parallel, Distributed and Network-Based Processing", 2011, DOI 10.1109/PDP.2011.32,pp. 141-145

[2] R.J. Hulsebosch, M.S. Bargh, G. Lenzini, P.W.G Ebben, and S.M. Iacob, "Context Sensitive Adaptive Authentication", Proc. of EuroSSC 2007, LNCS 4793, pp. 93-109.

[3] J. Brainard, A. Juels, R. Rivest, M. Szydlo, and M. Yung, "Fourth Factor Authentication: Somebody You Know", Proc. of ACM CCS 2006, pp. 168- 178

[4] H. Zheng, J. Kwak, K. Son, W. Lee, S. Kim, and D. Won, "Confidence Value Based Multi Levels of Authentication for Ubiquitous Computing Environments", Proc. of ICCSA 2006, LNCS 3981, pp. 954-963.

[5] B. Schneier, "Two-Factor Authentication: Too Little, Too Late", Communications of ACM, Vol. 48, No. 4, Apr. 2005, 136.

[6] M. Alexander, "Keeping Online Banking Safe: Why Banks Need Geolocation and Other New Techniques Right Now". http://www.bankersonline.com/security/safebanking.html , May 2005.

[7] Federal Financial Institutions Examination Council, "Authentication in Internet Banking Environment", http://www.ffiec.gov/press/pr101205.htm, Oct. 2005.

[8] E. Toye, R. Sharp, A. Madhayapeddy, and D. Scott, "Using Smart Phones to Access Site-Specific Services", IEEE Pervasive Computing, Springer- Verlag, Vol. 4, Issue 2, pp. 60-66, 2005.

[9] K. Nichols Randall and Panos C. Lekkas, "Wireless security: models, threats, and solutions", Tata McGraw Hill, 2006.
[10] F. Dominici, D. Mazzocchi, P. Mulassano, M. Spelat, G. Boiero, P. Lovisolo, "NAV/COM Hybrid Architecture for Innovative Location Based Payment Systems", Proc of CCNC 2009, pp. 1-5.

[11] D.E. Denning and P.F. MacDoran, "Location- based authentication: grounding cyberspace for better security", Computer Fraud \& Security, Vol. 1996, Issue 2, Feb. 1996, pp. 12-16.

[12] A.I. Gonz' alez-Tablas Ferreres, B. Ramos Alvarez, and A.R. Garnacho, "Guaranteeing the Authenticity of Location Information", IEEE Pervasive Computing, Vol. 7, Issue 3, July-Sept. 2008, pp. 72-80.

[13] M.G. Kuhn, "An Asymmetric Security Mechanism for Navigation Signals", Proc. of Sixth Int'l Workshop Information Hiding (IH) 2004, LNCS 3200, pp. 239-252.

[14] D. Qiu, "Security Analysis of Geoencryption: A Case Study Using Loran", Proc. of ION GNSS 2007, Texas, USA, Sept. 2007, pp. 1146- 1154

[15] S. M. Gaikwad, R. R. Joshi, and Mulay, P., "Cluster Mapping with the help of New Extended MCF Algorithm and MCF Algorithm to Recommend an Ice Cream to the Diabetic Patient," METHODOLOGY, vol. 1, p. 7.

[16] S. M. Gaikwad, R. R. Joshi, Mulay, P. (2015). Analytical Network Process (ANP) to Recommend an Ice Cream to a Diabetic Patient. IJCA Online 121 (12), 49-52.

[17] S. M. Gaikwad, R. R. Joshi, and Mulay, P., "System Dynamics Modeling for Analyzing Recovery Rate of Diabetic Patients by Mapping Sugar Content in Ice Cream and Sugar Intake for the Day," in Proceedings of the Second International Conference on Computer and Communication Technologies, ed: Springer India, 2016, pp. 743-749.

[18] S. M. Gaikwad, Mulay, P., and R. R. Joshi, " Mapping with the help of new Proposed Algorithm and Modified Cluster Formation Algorithm to recommend an Ice Cream to the Diabetic Patient based on Sugar Contain in it," International Journal of Students' Research in Technology \& Management, vol. 3, pp. 410-412, 2015.

[19] Suhas Gaikwad: Cohort Intelligence and Genetic Algorithm along with AHP to recommend an Ice Cream to a Diabetic Patient. Lecture Notes In Computer Science, 12/2015: chapter Cohort Intelligence and Genetic Algorithm along with AHP to recommend an Ice Cream to a Diabetic Patient: pages 1-9; SEMCCO 2015.

[20] Suhas Gaikwad: Cohort Intelligence and Genetic Algorithm along with Modified Analytical Hierarchy Process to Recommend an Ice Cream to a Diabetic Patient. Advances in Intelligent Systems and Computing, 03/2016: chapter Cohort Intelligence and Genetic Algorithm along with Modified Analytical Hierarchy Process to Recommend an Ice Cream to a Diabetic Patient: pages 1-8; ICDECT. ISBN: ISSN: 2194-5357

[21] S. M. Gaikwad, R. R. Joshi, Mulay, P., "Attribute visualization and cluster mapping with the help of new proposed algorithm and modified cluster formation algorithm to recommend an ice cream to the diabetic patient based on sugar contain in it," vol. 10, pp. 1-6, 2015. 
[22] S. M. Gaikwad, "Cluster mapping with the help of new proposed algorithm and MCF algorithm to recommend an ice cream to the diabetic Patient," International Journal of Applied Engineering Research, vol. 10, pp. 21259- 21266, 2015.

[23] S. M. Gaikwad, R. R. Joshi, Mulay, P. "Modified Analytical Hierarchy Process to Recommend an Ice Cream to a Diabetic Patient," pp. 1-6, 2015.

[24] Gaikwad, S. M., Mulay, P., \& Joshi, R. R. (2015). Analytical Hierarchy Process to Recommend an Ice
Cream to a Diabetic Patient Based on Sugar Content in it. Procedia Computer Science, 50, 64-72.

[25] M. Spelat and F. Margary, "GAL-PMI Project: Global Navigation Satellite Systems to Support Mobility and Security", Proc. of Space Applications Days 2008, Toulouse (France), 22-25 April 2008, pp. 608- 612. 